$\xi=$ 줄

\title{
Occurrence of diversity in dental pattern and their role in identification in Udaipur population: an orthopantomogram based study
}

\author{
Rashmi Metgud ${ }^{1 *}$, Bhardwaj Tina Neelesh ${ }^{2}$, Smitha Naik ${ }^{3}$, Shrikant Patel ${ }^{4}$ \\ ${ }^{1}$ Prof. \& HOD, Oral \& Maxillofacial Pathology and Microbiology, Pacific Dental College \& Hospital, Udaipur \\ ${ }^{2}$ Post Graduate Student, Oral \& Maxillofacial Pathology and Microbiology, Pacific Dental College \& Hospital, Udaipur \\ ${ }^{3}$ Reader, Oral \& Maxillofacial Pathology and Microbiology, Pacific Dental College \& Hospital, Udaipur \\ ${ }^{4}$ Senior Lecturer, Oral \& Maxillofacial Pathology and Microbiology, Pacific Dental College \& Hospital, Udaipur \\ *Corresponding author E-mail:rashmi_metgud@rediffmail.com
}

\begin{abstract}
Background: Dental comparison is based on unique characteristics of the teeth (shape and outline, restorations, supernumerary teeth, impacted, fractured teeth etc.). Being the hardest structure in human body, teeth represent an ideal means of identification in situations of advanced decomposition, fire and mass disaster. Orthopantomogram is a broadly applied standard method in today's practical dentistry and provides a complete view of the teeth and both jaws in one image.

AIM: The present study aimed to verify the diversity of dental patterns in Udaipur population based on specific patterns of missing, filled, unrestored (virgin) and impacted teeth using Orthopantomogram.

Material and Methods: 300 Dental Orthopantomogram were randomly selected from Pacific Dental College \& Hospital, Udaipur and observed for the occurrence of dental patterns. The frequency of occurrence of dental patterns and the diversity in dental patterns were calculated for full dentition, maxilla and mandible.

Results: Occurrence of most common dental pattern was formed by 32 virgin teeth, $10.66 \%$. the diversity of dental pattern for full dentition was $99.7 \%$, maxilla was $99.5 \%$, and mandible was $99.49 \%$.

Conclusion: The diversity derived from dental patterns is a potentially valuable tool with broad applicability for human identification inspite of the mentioned drawbacks.
\end{abstract}

Keywords: Diversity; Dental Patterns; Identification; Orthopantomogram

\section{Introduction}

Fingerprinting is the most accurate method of identification of people. But in cases of disfigured, decomposed, burnt or fragmented bodies, it is of limited value. Teeth being the hardest structures of the human body and, as such, represent an ideal means of identification in these situations as they retain a number of their original characteristics, which are often unique and hence offer a possibility of rather accurate and legally acceptable identification of such remains.(Singh et al. 2013)

Studies at the University of Tennessee, Knoxville, have shown that soft tissue decay can be complete in as short as two weeks after death, making visual identification or fingerprint comparison impossible. Regardless of the condition of the body, it is very likely that the teeth will be preserved, and it is this line of evidence that oftentimes proves to be the most reliable comparative tool.(Adams 2003)

Forensic dental identification of human remains largely depends upon the comparison of post-mortem dental characteristics to ante-mortem dental information such as written records, dental casts and radiographs. With recent advances in dental investigations and a more streamlined approach to practicing dentistry, dental records such as periapical radiographs, panoramic radiographs, treatment notes, dental charts, dental casts and intraoral photographs are commonly applied methods of personal investigations and post mortem and ante mortem comparisons. Dental radiographs are certainly one of the most desirable pieces of antemortem evidence because of their highly objective nature as compared with other records.(Kumar et al. 2014)

Gustafson was the first to use Orthopantomography (OPG) in forensic practice for identification. The availability of OPGs has established this dental record as a valuable aid in comparing antemortem and post mortem dental characteristics. OPG Provide a complete view of both jaws and teeth in one image and offer advantages in terms of dental record keeping. Therefore, it can be applied more economically and practically in identifying a large number of victims from mass disasters and other calamities.(Kumar et al. 2014)

Adams reported upon the diversity of adult dental patterns using empirical observations of large reference data sets and showed the very diversity. These reports were specifically related to nonradiographic dental comparison and the diversity was analyzed based on them. Compared to dental charts, which may be subjective, dental radiographs are more objective and show relatively less errors. In addition, since post-mortem investigators can evaluate ante-mortem and post-mortem radiographs of dental charts which carry the risk of errors among the different investigators. For these reasons there is a clear need for the diversity of dental 
patterns in Orthopantomograms to be explored in more detail.(Lee et al. 2004)

Dental comparison is based on unique characteristics of the teeth (shape, outline, restorations, supernumerary teeth, impacted teeth, fractured teeth etc.). The vast number of possible combinations of these characteristics in human dentition, can give rise, in theory, to trillions of possible dental patterns that could allow the identification process to be quantified as reported. By empirically observing the frequencies of dental patterns from large representative datasets, it is possible to estimate accurately the diversity of the population as a whole.(Kumar et al. 2014)

The present study aimed for further research in the field of post mortem identification of especially mass disaster and tragedies to identify the diversity of dental pattern in the Udaipur population based on specific patterns of missing, filled, unrestored and impacted teeth using OPGs.

\section{Material and methods}

The present study was outlined with a view to study the larger databases as an easy and feasible way of keeping records for identification. 300 OPGs were randomly selected which were stored at Pacific Dental College and Hospital, Udaipur. In each OPG, each tooth was observed and was then coded and categorized into Virgin (V), Restored (R), Missing (M) and Impacted (I) teeth. [TABLE 1].(Kumar et al. 2014)

Table 1: Dental Codes on the Orthopantomogram

\begin{tabular}{|c|c|c|}
\hline Code & $\begin{array}{l}\text { Tooth Desig- } \\
\text { nated }\end{array}$ & Description \\
\hline $\mathrm{V}$ & Virgin tooth & No evidence of dental treatment, decaying \\
\hline $\mathrm{R}$ & Restored tooth & $\begin{array}{l}\text { Amalgam, composite or any other restoration, } \\
\text { single unit crowns etc. }\end{array}$ \\
\hline M & Missing tooth & Radiographically missing teeth \\
\hline I & Impacted tooth & Unerupted or impacted tooth \\
\hline
\end{tabular}

The data collected from the radiograph formed the dental pattern of that particular individual which was distinct to that particular individual. Only permanent teeth were considered. Dentulous and partially edentulous subjects were included for analysis. All OPGs with cyst, tumors or any maxillofacial abnormality were excluded from the study as they would itself be a distinct characteristic of that particular individual. Each dental arch (maxilla and mandible) on OPG was sectioned into 6 segments. [TABLE 2]. The OPGs were thus analyzed and the occurrence of diversity of dental patterns were calculated for full dentition, maxilla and mandible.

Table 2: Sections of Dental Arches

\begin{tabular}{ll}
\hline Maxillary right posterior teeth & $14-18$ \\
\hline Maxillary anterior teeth & $13-23$ \\
Maxillary left posterior teeth & $24-28$ \\
Mandibular right posterior teeth & $34-38$ \\
Mandibular anterior teeth & $33-43$ \\
Mandibular left posterior teeth & $44-48$ \\
\hline
\end{tabular}

\section{Statistical analysis}

The data was analyzed using Statistical Package For Social Sciences (SPSS) 19.0 version software. The test applied was descriptive statistics.

\section{Results}

The most common dental pattern observed was formed by the combination of 32 virgin teeth, $10.66 \%$ in the sample. [TABLE 3] In the analyzed sample, number of different dental patterns and number of individual dental patterns which occurred only once in the sample were calculated. [TABLE 4]

The diversity was empirically calculated as per SS Lee 2004.(Lee et al. 2004).
Table 3: Occurrence of Five Most Commonly Observed Dental Patterns

\begin{tabular}{lll}
\hline Dental Pattern & Frequency & Percentage \\
\hline VVVVV VVVVVV VVVVV & 32 & 10.66 \\
VVVVV VVVVVV VVVVV & & 4 \\
IVVVV VVVVVV VVVVI & 12 & 4.33 \\
IVVVV VVVVVV VVVVI & & \\
VVVVV VVVVVV VVVVV & 10 & 1 \\
IVVVV VVVVVV VVVVI & & \\
IVVVV VVVVVV VVVVI & 4 & 1 \\
VVVVV VVVVVV VVVVV & & \\
VVVVV VVVVVV VVVVI & 4 & \\
IVVVV VVVVVV VVVVI & 4 &
\end{tabular}

Table 4: Occurrence of Dental Patterns in Full Dentition, Maxilla, Mandible

\begin{tabular}{lll}
\hline Dentition & $\begin{array}{l}\text { No. Of Dental Pat- } \\
\text { tern }\end{array}$ & $\begin{array}{l}\text { No. Of Individual Dental Pat- } \\
\text { terns }\end{array}$ \\
\hline $\begin{array}{l}\text { Full Denti- } \\
\text { tion }\end{array}$ & 235 & 220 \\
Maxilla & 150 & 129 \\
Mandible & 148 & 131 \\
\hline
\end{tabular}

Diversity in dental patterns for full dentition was $99.7 \%$, maxilla was $99.5 \%$, and mandible was $99.49 \%$.

\section{Discussion}

Forensic odontology is the branch of forensic medicine which in the interest of justice deals with the proper handling and examination of dental evidence and with the proper evaluation and presentation of the dental findings.(Kumar et al. 2014) OPG or panoramic radiograph is the most commonly preferred extra-oral dental radiograph by dentists and it also provides a wide coverage of both the jaws and teeth. OPG expands the spectrum of forensic radiology for the individual case and can also offers a reliable aid for victim identification in the wake of mass disasters, aircraft crashes and terrorist attacks, where a large number of bodies have to be identified under great pressure.(Kumar et al. 2013) One of the greatest challenges of dental identification is having the largest number of similarities and the smallest number of discrepancies in order to obtain a positive identification in ante-mortem and postmortem records.

In the present study, the average time taken for manual coding of all the six sextants of an OPG was 2 minutes 30 seconds. The diversity of dental pattern calculated for full dentition, maxilla as well as for mandible was in consonance with the study conducted by SS Lee 2004. Also, the diversity of dental patterns in full dentition in the present study was in accordance with the study conducted by, Abhishek Kumar et al. 2014, however the values for maxilla and mandible differed greatly.

The high value of diversity for full dentition, maxilla and mandible implies sufficient power for personal identification. For example, the probability of finding a subject with missing third molars in the upper right and left teeth region and missing third and second molars in the lower quadrant with the entire rest teeth being virgin can be as less as $1 \%$ in a given sample. The four parameters which were used in the present study were primarily for specific implementation in the Indian scenario. Dental caries was excluded as a parameter for dental diversity as it is a slow progressing disease and its extent cannot be identified. Furthermore, initial radiolucency of the carious lesion can be misinterpreted with the artifact of the radiograph.(Lee et al. 2004)

Sweet points out that one of the greatest difficulties in using the method of diversity of dental patterns is the availability of antemortem dentistry data. In developing countries, like India, the main reason individuals are not identified is the absence of data or lack of access to dental services. Another reason is that records are not kept for long time. Under article $51 \mathrm{~A}(\mathrm{~h})$ of the constitution of India, there is a moral obligation on the doctor, and a legal duty, to maintain and preserve medical, medico-legal, and legal documents in the best interests of social and professional justice. According to recent surveys the practice of dental record maintenance is surprisingly low in India.(Biazevic et al. 2011) 
Diversity of dental patterns formed by combinations of missing, filled, and unrestored teeth can be comparable to the diversity of mitochondrial DNA ( $\mathrm{mtDNA}$ ) sequences formed by combinations of variants at multiple polymorphic sites within the mtDNA sequence. mtDNA provides an excellent frame of reference in comparison with dental patterns.(Biazevic et al., 2011) However, the lower stability of dental pattern in the population unlike DNA which is only affected by mutation and heteroplasmy can be a disadvantage at times.(Lee et al. 2004)

\section{Conclusion}

The dental records can play a primary source of identification in spite of the advances in DNA identification technology. However, the results of the present study are to be used with 'caution' and more researches with a larger sample size from randomly distributed samples are necessary. One useful step in this direction would be to develop large, periodically updated dental pattern datasets for different populations, and more importantly, to pool as many of these datasets as possible since typical forensic scenarios will involve people from different countries and population. Despite the mentioned drawback of the identification method, dental information may be in several cases the only available means of identification, where most of the other popular methods of identification are completely destroyed.

\section{References}

[1] Adams, B. J (2003) Establishing personal identification based on specific patterns of missing, filled, and unrestored teeth. Journal of forensic sciences, 48, 487-496. http://dx.doi.org/10.1520/JFS2002226.

[2] Biazevic, M. G. H., De Almeida, N. H., Crosato, E. \& MichelCrosato, E (2011) Diversity of dental patterns: Application on different ages using the Brazilian National Oral Health Survey. Forensic science international, 207, 240. E1-240. e9.

[3] Kumar, A., Ghosh, S. \& Logani, A (2014) Occurrence of diversity in dental pattern and their role in identification in Indian population: An orthopantomogram based pilot study. Journal of forensic dental sciences, 6, 42. http://dx.doi.org/10.4103/0975-1475.127770.

[4] Kumar, N., Sreenivasan, V., Patil, P. \& Vashishth, S (2013) Panoramic Imaging as a Tool of Identification in Forensic Odontology. Indian Journal of Forensic Odontology, 6, 51 .

[5] Lee, S.-S., Choi, J.-H., Yoon, C.-L., Kim, C.-Y. \& Shin, K.-J (2004) the diversity of dental patterns in the orthopantomography and its significance in human identification. Journal of forensic sciences, 49, 784-786. http://dx.doi.org/10.1520/JFS2003339.

[6] Singh, S., Bhargava, D. \& Deshpande, A (2013) Dental orthopantomogram biometrics system for human identification. Journal of forensic and legal medicine, 20, 399-401. http://dx.doi.org/10.1016/j.jflm.2013.02.001. 